\title{
MONADS DEFINED BY INVOLUTION-PRESERVING ADJUNCTIONS
}

BY

\author{
PAUL H. PALMQUIST
}

\begin{abstract}
Consider categories with involutions which fix objects, functors which preserve involution, and natural transformations. In this setting certain natural adjunctions become universal and, thereby, become constructible from abstract data. Although the formal theory of monads fails to apply and the Eilenberg-Moore category fails to fit, both are successfully adapted to this setting, which is a 2-category. In this 2-category, each monad ( $=$ triple $=$ standard construction) defined by an adjunction is characterized by a pair of special equations. Special monads have universal adjunctions which realize them and have both underlying Frobenius monads and adjoint monads. Examples of monads which do (respectively, do not) satisfy the special equations arise from finite monoids (= semigroups with unit) which are (respectively, are not) groups acting on the category of linear transformations between finite dimensional Euclidean (= positive definite inner product) spaces over the real numbers. More general situations are exposed.
\end{abstract}

This paper adapts the Eilenberg-Moore category [1], [4, p. 136] and a formal generalization [7] to a setting (categories with involution) where the ordinary construction is not appropriate. For example, to construct Euclidean (= positive definite inner product vector) spaces over the complex numbers $\mathbf{C}$ from those over the real numbers $\mathbf{R}$, we select special Eilenberg-Moore algebras for a monad (= triple) on the category $\mathbf{E}(\mathbf{R})$ of $\mathbf{R}$-linear transformations between Euclidean spaces over $\mathbf{R}$, where the monad $\mathbf{T}$ is defined by the adjunction corresponding to the forgetful functor $\mathbf{E}(\mathbf{C}) \rightarrow \mathbf{E}(\mathbf{R})$. "Externally", the specialalgebras are those in the image of the comparison functor $K: \mathrm{E}(\mathrm{C}) \rightarrow \mathrm{E}(\mathrm{R})^{\mathrm{T}}$; thus an algebra fails to be special if scalar multiplication by $z \in \mathbf{C}$ with modulus $|z|=1$ does not preserve distance, for example, the algebra $(\mathbf{R} \oplus \mathbf{R}, h)$ with action $i \cdot(a, b)=(a+2 b,-a-b)$ fails. Hence, the adjunction for $\mathbf{E}(\mathbf{C}) \rightarrow \mathbf{E}(\mathbf{R})$ is not monadic (= tripleable), that is, not terminal with respect to all adjunctions defining the monad $\mathbf{T}$. Fortunately, the adjunction is terminal in the appropriate

Received by the editors September 23, 1974.

AMS (MOS) subject classifications (1970). Primary 18C15, 18D99, 18A40, 18D05; Secondary 20C99, $20 \mathrm{M} 30$.

Key words and phrases. Category with involution, monad, adjunction, 2-category, *-monad, Eilenberg-Moore category, Kleisli category, $2^{\circ}$-category, adjoint monad, Frobenius monad, *-category. 
setting which consists of categories with involutions fixing objects and of functors preserving involutions, and moreover, special algebras have an "internal" description depending on a special property (*) of each monad defined by an involution-preserving adjunction.

In $\S 1$ we describe this restricted setting axiomatically as the $2^{\circ}$-category *Cat, in which adjunctions define monads characterized by a pair of (additional) equations (*), and in which only these *-monads have (universal) realizations. \$2 shows that each finite group yields a *-monad on certain categories via a natural construction taking each monoid (= semigroup with unit) into a monad. The *algebras for these *-monads are (unitary) representations of the group. \$3 generalizes $\S 1$ to enriched categories over a suitable closed category. $\$ 4$ gives a necessary adaptation of [7] which then yields analogous formal results. $\$ 5$ relates *-monads to Frobenius monads [3, pp. 151-152] and to adjoint monads [1, pp. 387-388] and gives conceptual descriptions of the involution on the Eilenberg-Moore category and of $*$-monads. $\$ 6$ contains further examples of *-monadic adjunctions.

We assume the reader is familiar with the prime example of a 2-category which is the (2-dimensional) category of all (0) categories, (1) functors and (2) natural transformations [4, pp. 42-44]. The definitions in it of adjunction [4, p. 81], $\langle F, U, \eta, \epsilon\rangle: X \rightarrow A$ and of monad [4, p. 133], $\mathrm{T}=\langle T, \eta, \mu\rangle$ generalize to any 2-category; in detail, an adjunction consists of a pair of 0-cells $\mathbf{X}, \mathbf{A}, \mathbf{a}$ pair of 1-cells $F: \mathrm{X} \rightarrow \mathrm{A}, U: \mathbf{A} \rightarrow \mathbf{X}$ and a pair of 2-cells $\eta: 1 \rightarrow U F, \epsilon: F U \rightarrow$ 1 which satisfy the triangular identities $\epsilon F \cdot F \eta=F$ and $U \epsilon \cdot \eta U=U$; while a monad $\mathrm{T}$ consists of a 0-cell $\mathrm{X}$, a 1-cell $T: \mathrm{X} \rightarrow \mathrm{X}$ and a pair of 2-cells $\eta: 1 \rightarrow T$, $\mu: T T \rightarrow T$ (called the unit and the multiplication of the monad) which satisfy the unit laws $\mu \cdot T \eta=\mu \cdot \eta T=1$ and the associative law $\mu \cdot \mu T=\mu \cdot T \mu$. Similarly, each adjunction $J=\langle F, U, \eta, \epsilon\rangle$ defines a monad $\mathrm{M}(J)=\langle U F, \eta, U \epsilon F\rangle$.

1. Equations and universal realizations in $2^{\circ}$-categories. For precision in stating the characterizing equations, the universal properties, and the various realizations, we augment the language of 2-categories as follows. Define a $2^{\circ}$-category $\left\langle T,()^{\circ}\right\rangle$ as a 2-category $T$ equipped with a weakly involutory 2 -functor ()$^{\circ}$ which is the identity on 0 -cells and 1-cells, but reverses each 2-cell $\theta$ and satisfies $\left(\theta^{\circ}\right)^{\circ}=$ $\theta$. Define a *-monad in $T$ as a monad $\langle T, \eta, \mu\rangle$ satisfying the (additional) equations

$$
\eta^{\circ} T \cdot \mu T \cdot T \mu^{\circ}=\mu \text { and } T \eta^{\circ} \cdot T \mu \cdot \mu^{\circ} T=\mu .
$$

Proposition 1 . In the 2-category underlying $a 2^{\circ}$-category, each adjunction yields a *-monad.

Proof. Given such an adjunction $\langle F, U, \eta, \epsilon\rangle$, construct the monad 
$\langle U F, \eta, U \epsilon F\rangle$. The first equation of (*) follows from the functorality of composition and the triangular identity for adjunctions:

$$
\eta^{\circ} U F \cdot U \epsilon F U F \cdot U F U \epsilon^{\circ} F=\eta^{\circ} U F \cdot U \epsilon^{\circ} F \cdot U \epsilon F=U F \cdot U \epsilon F=U \epsilon F .
$$

A similar argument verifies the second equation of (*).

The converse holds for the $2^{\circ}$-categories *Cat and I cat described below (and other examples in §3). Recall that the 2-category Cat has categories as 0 cells, functors as 1-cells, and natural transformations as 2-cells. Let an I-category $* X=\left(X,()^{*}\right)$ be a category $\mathbf{X}$ equipped with an involution functor

$$
()^{*}: \mathbf{X}^{\text {op }} \rightarrow \mathbf{X}, \quad\left(f^{*}\right)^{*}=f \text { for } f \text { in } \mathbf{X} \text {; }
$$

let an I-functor $F: * X \rightarrow * Y$ be an involution-preserving functor $F\left(f^{*}\right)=(F f)^{*}$; and let an I-natural transformation $\theta$ be just a natural transformation. This yields a 2-category $I$ cat and a faithful, forgetful 2-functor $I$ cat $\rightarrow$ Cat. For a 2-cell $\theta$ in $I \operatorname{cat}(* X, * Y)$ the equation

$$
\left(\theta^{\circ}\right)_{X}=\left(\theta_{\left(X^{*}\right)}\right)^{*} \text { for } X \in \mathbf{X}
$$

defines the weak involution making $I$ cat a $2^{\circ}$-category.

Similarly, letting a *-category $* \mathbf{X}$ be an I-category with each object $X$ fixed by involution $X^{*}=X$, we obtain a $2^{\circ}$-category $*$ Cat and an inclusion $2^{\circ}$-functor $*$ Cat $\rightarrow I$ cat; for $*$-categories $* X$ and $* Y$ we have the ()$^{\circ}$-preserving equality of hom-categories

$$
* \operatorname{Cat}(* X, * Y)=I \operatorname{cat}(* X, * Y) \text {. }
$$

THEOREM 2. In each of the 2-categories * Cat and Icat, each *-monad has both a terminal realization and an initial realization.

CoROllary 3. Being a *-monad is a necessary and sufficient condition for a monad to have a realization in * Cat or $I$ cat.

Proof of Theorem 2. The statement of universal properties is analogous to that for the adjunctions in Cat constructed by Eilenberg-Moore [1] , [4, p. 138] and by Kleisli [2], [4, pp. 143-144]; our constructions augment theirs and will be described in terms of theirs.

First, in $*$ Cat given a $*$-monad $T=\langle T, \eta, \mu\rangle$ on $* \mathrm{X}$, we describe the terminal realization

$$
J=\langle F, U, \eta, \epsilon\rangle: * \mathrm{X} \rightarrow * \mathrm{X}^{S \mathrm{~T}} \text { in } * \text { Cat }
$$

via the terminal (Eilenberg-Moore) realization

$$
J_{1}=\left\langle F_{1}, U_{1}, \eta_{1}, \epsilon_{1}\right\rangle: X \rightarrow \mathbf{X}^{\mathbf{T}} \text { in Cat. }
$$


The canonical comparison functor $\iota: X^{S T} \rightarrow X^{T}$ in Cat is the full inclusion of those T-algebras $\langle X, h: T X \rightarrow X\rangle$ fixed by the (proposed) involution

$$
\langle X, h\rangle \mapsto\left\langle X, \eta_{X}^{\circ} \cdot \mu_{X} \cdot T h^{*}\right\rangle .
$$

Verification of the associative law for the new structure map requires the first equation (*) in addition to the associative laws both for the multiplication $\mu$ and for the original structure map $h$, while verification of the unit law requires only the unit laws both for the monad and for $h$. Via this involution, $\iota$ is an I-functor and $J_{1}$ is an adjunction in I cat. (Although we omit details here, a conceptual description of the involution is sketched in $\S 5$.) Thus from $\mathbf{X}^{\mathbf{T}}$ together with the involution, we obtain $* \mathrm{X}^{S T}$.

The terminal property of $J$ in $*$ Cat follows from that of $J_{1}$ in Cat. Given an adjunction

$$
J_{2}=\left\langle F_{2}, U_{2}, \eta_{2}, \epsilon_{2}\right\rangle: * \mathrm{X} \rightarrow * \mathbf{A} \text { in } * \text { Cat }
$$

realizing $\mathrm{T}$, take the unique (comparison) functor $K_{1}: \mathrm{A} \rightarrow \mathrm{X}^{\mathrm{T}}$ in Cat satisfying the equations

$$
K_{1} F_{2}=F_{1}, \quad U_{2}=U_{1} K_{1} \text { and } K_{1} \epsilon_{2}=\epsilon_{1} K_{1} .
$$

Verify that $K_{1}$ is an I-functor and factors as $K_{1}=K \iota$ for a unique $*$-functor $K$ : $* \mathbf{A} \rightarrow * \mathrm{X}^{S \mathrm{~T}}$. This comparison $*$-functor $K$ exhibits the terminal property of the realization $J$ in *Cat,

$$
K F_{2}=F, \quad U_{2}=U K \text { and } K \epsilon_{2}=\epsilon K .
$$

Second in I cat, the terminal realization of a $*$-monad has a shorter construction, but requires more "bookkeeping" to keep track of the involution on objects; the underlying category is the entire Eilenberg-Moore category, but the involution is now written as

$$
\langle X, h\rangle \mapsto\left\langle X^{*},\left(\eta_{X}\right)^{*} \cdot \mu_{\left(X^{*}\right)} \cdot T h^{*}\right\rangle .
$$

The terminal property in I cat follows directly from that in Cat.

Third, the initial realization in $*$ Cat is just Kleisli's Cat-realization equipped with the necessary involution, or, note that the free-algebras in the EilenbergMoore category are fixed by our involution.

Finally, the initial realization in I cat is obtained similarly.

COROllary 4. Consider a category supporting both a monad and an involution. The Eilenberg-Moore category of algebras can be equipped with an involution preserved by the adjunction if and only if the monad satisfies the equations (*). 
Such an adjunction can be lifted from Cat to I cat. However, in contrast, it cannot be lifted to $*$ Cat unless it is trivial, for example, the identity adjunction.

2. Group representations by *-monads. The following generalization of (finite) orthogonal direct sum is used. A *-category $* \mathbf{X}$ has finite orthogonal copowers, if $\mathrm{X}$ has a zero object and for each object $X$ and each finite set $I$, we have a coproduct $\left\{u_{i}: X \rightarrow{ }^{I} X \mid i \in I\right\}$ such that $\left(u_{i}^{*}\right) u_{j}=\delta_{i j}$ for all $i, j$ in $I$, where the Kronecker delta $\delta_{i j}$ equals 1 (the identity map) if $i=j$, and equals zero otherwise. Call $* \mathbf{X}$ nontrivial if (the identity map for) some object is not zero.

Proposition 5. Consider a $*$-category $* \mathrm{X}$ with finite orthogonal copowers. Each finite monoid (in sets) yields a monad on $\mathrm{X}$, but if $* \mathrm{X}$ is nontrivial, only groups yield *-monads.

Proof. Given a monoid $\langle M, e, \cdot\rangle$, construct the monad

$$
\mathrm{T}=\left\langle X \mapsto{ }^{M} X, u_{e}, \mu_{X}={ }^{(\bullet)} X:{ }^{M \times M_{X}} \rightarrow{ }^{M} X\right\rangle .
$$

It is well known that the monad laws follow from the monoid laws.

Assume that the monad $\mathrm{T}$ satisfies the first equation of (*) and that the object $X$ has $1 \neq 0$. Since we identify ${ }^{M}\left({ }^{M} X\right)={ }^{M} \times{ }^{M} X$, the multiplication $\mu$ is determined by the equation

$$
\left(u_{a}\right)^{*} \cdot \mu \cdot u_{(b, c)}=\delta_{a, b \cdot c} \text { for all } a, b, c \in M .
$$

Fixing $a=c \in M$ and $b=e$, we apply the function $\mathrm{X}\left(u_{(e, a)},\left(u_{a}\right)^{*}\right)$ to both sides of the first equation of $(*)$, and obtain

$$
\begin{aligned}
\left(u_{a}\right)^{*} \cdot \mu \cdot u_{(e, a)} & =\left(u_{a}\right)^{*} \cdot T \eta^{\circ} \cdot T \mu \cdot\left(\mu^{\circ}\right) T \cdot u_{(e, a)}, \\
\delta_{a, e \bullet a} & =\left[\left(u_{(a, e)}\right)^{*} \cdot T \mu\right] \cdot\left[\left(\mu^{\circ}\right) T \cdot u_{(e, a)}\right], \\
1 & =m \cdot n,
\end{aligned}
$$

where $m=\left(u_{(a, e)}\right)^{*} \cdot T \mu$ and $n=\left(\mu^{\circ}\right) T \cdot\left(u_{(e, a)}\right)$. Since $1 \neq 0$, we have $m \neq 0$ and $n \neq 0$. Using properties of coproducts and products, we see there must exist $x, y \in M$ such that $m \cdot u_{(a, x, a)} \neq 0$ and $\left(u_{(a, y, a)}\right)^{*} \cdot n \neq 0$, and hence $e=x \cdot a$ and $e=a \cdot y$. Thus each element has an inverse, and $M$ was $a$ priori a group.

Verifying that groups yield *-monads is essentially the above in reverse. We omit further details since the special case when $\mathbf{X}$ is additive has been studied by Lawvere [3, p. 152] for the related concept of Frobenius monad (see our §5).

This proposition generalizes to I-categories with finite bipowers, where for each object $X$ and finite set $I$ we have a pair of coproducts $\left\{u_{X, i}: X \rightarrow{ }^{I} X \mid i \in I\right\}$ and $\left\{u_{X^{*}, j}: X^{*} \rightarrow{ }^{I}\left(X^{*}\right) \mid j \in I\right\}$ such that $\left({ }^{I} X\right)^{*}={ }^{I}\left(X^{*}\right)$ and $\left(u_{X, i}\right)^{*} u_{X^{*}, j}=\delta_{i j}$. The proof involves a little more notational bookkeeping. 
3. Enrichment over closed categories. We may generalize from categories (based on sets) to enriched categories over a closed category $V$ [4, pp. 180-181]. The 2-category $V$-Cat replaces $C$ at. Since $V$ is symmetric each $V$-category $\mathbf{X}$ has an opposite category $X^{o p}$. Hence, we may define the 2-categories I-V -Cat and *-V-Cat with underlying 2-functors forming the commutative diagram

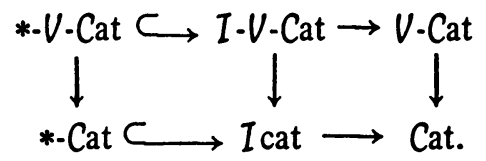

The left-hand square is, moreover, a commutative square of $2^{\circ}$-categories and $2^{\circ}$. functors.

All statements in $\$ 1$ about I cat and $*$ Cat hold for their enriched versions I-V-Cat and $*-V$-Cat, except the existence of the terminal realizations may depend on their existence in $V$-Cat, hence on the existence of certain equalizers in $V$.

4. Formal theory of *-monads. As the development of the formal theory of $*$-monads parallels that of monads [7], we only indicate our point of departure. For each $2^{\circ}$-Cat $T$ we construct a $2^{\circ}$-category $* \operatorname{Mnd}(T)$ related by the inclusion $2^{\circ}$-functor $* \operatorname{Inc}_{T}: T \rightarrow * \operatorname{Mnd}(T)$ and its left $2^{\circ}$-adjoint which is the underlying $2^{\circ}$-functor $*$ Und $_{T}: * \operatorname{Mnd}(T) \rightarrow T$. We say the $2^{\circ}$-category $T$ admits the construction of algebras with involution, if the inclusion $2^{\circ}$-functor has a right $2^{\circ}$. adjoint $* \operatorname{Alg}_{T}: * \operatorname{Mnd}(T) \rightarrow T$. Then formal consequences can be derived as with monads. Note that *Cat and I cat admit the construction of algebras with involution. Without the last two words, the definition is Street's and the statement is false, since not all monads in $*$ Cat have realizations.

In more detail, the 0 -cells of $* \operatorname{Mnd}(T)$ are $*$-monads $T$ in $T$. Recall $\operatorname{Mnd}(T)$ denotes the 2-category of monads, monad functors, and monad functor transformations [7]. For *-monads, $\mathbf{S}$ and $\mathbf{T}$ in $T$, there is the full inclusion of categories

$$
* \operatorname{Mnd}(T)(S, T) \subset \operatorname{Mnd}(T)(S, T)
$$

determined by the monad functors $(U, \phi): \mathrm{S} \rightarrow \mathrm{T}$ which are fixed by the involution

$$
(U, \phi) \mapsto\left(U,\left(\eta^{\circ} \cdot \mu\right) U T \cdot S \phi^{\circ} T \cdot S U\left(\mu^{\circ} \cdot \eta\right)\right) .
$$

A more conceptual description of the involution can be derived from $\S 5$.

Formal results on the existence of generators for a *-monad, the comparison theorem and monadicity theorems follow as in [7].

5. A conceptual characterization of *-monads and the involution on their Eilenberg-Moore categories. First, we analyze the involution constructed in 
Theorem 2. Starting with an adjoint monad $\mathbf{T} \rightarrow \mathbf{G}[1, \mathrm{pp} .387-388]$ on a category with involution $\left(\mathrm{X},()^{*}\right)$, we have the composite isomorphism

$$
p:\left(\mathrm{X}^{\mathrm{T}}\right)^{\mathrm{op}} \stackrel{(a)^{\mathrm{op}}}{\longrightarrow}\left({ }^{\mathrm{G}} \mathrm{X}\right)^{\mathrm{op}} \stackrel{f}{\longrightarrow}\left(\mathrm{X}^{\mathrm{op}}\right)^{\left(\mathrm{G}^{\mathrm{op}}\right)} \stackrel{()^{*}}{\longrightarrow} \mathrm{X}^{\left(\mathrm{G}^{\circ}\right)}
$$

where the isomorphism $a$ between the category of $\mathrm{T}$-algebras and the category of G-coalgebras was exposed by Eilenberg-Moore [1, p. 389], the next isomorphism $f$ is clear from the usual construction of coalgebras or can be deduced formally $[7, \text { p. 164], and the final isomorphism comes via the involution ( })^{*}: X^{\text {op }} \rightarrow \mathbf{X}$ by extending our notation $\mathbf{G}^{\circ}=()^{*} \mathbf{G}^{\mathrm{op}}()^{*}$. Since this yields the adjoint monad $\mathbf{G}^{\circ} \dashv \mathbf{T}^{\circ}$, we may iterate the displayed isomorphism $p$ and verify that it has "period 2". Thus $p$ is an involution on $\mathbf{X}^{\mathbf{T}}$, if the monad $\mathbf{G}^{\circ}$ equals $\mathbf{T}$. The latter property is clear for the adjoint monad underlying a *-monad as described below.

Proposition 6. Each *-monad has an underlying Frobenius monad, and hence, an underlying adjoint monad.

Proof. Lawvere [3, pp. 151-152] calls the data $\langle F, \eta, \mu, \epsilon, \delta\rangle$ a Frobenius monad if $\langle F, \eta, \mu\rangle$ is a monad, $\langle F, \epsilon, \delta\rangle$ is a comonad, and they satisfy the four equations

$$
\begin{array}{ll}
\epsilon F \cdot \mu F \cdot F \delta=\mu, & F \epsilon \cdot F \mu \cdot \delta F=\mu, \\
F \mu \cdot \delta F \cdot \eta F=\delta, & \mu F \cdot F \delta \cdot F \eta=\delta .
\end{array}
$$

Given a *-monad $\langle S, \eta, \mu\rangle$, take the data $\left\langle S, \eta, \mu, \eta^{\circ}, \mu^{\circ}\right\rangle$. The first two conditions for a Frobenius monad are satisfied, while the first two equations are just equations (*) and the last two are involutes of (*).

We omit the straightforward verification of Lawvere's remark that Frobenius monads yield adjoint monads. For the *-monad $S=\langle S, \eta, \mu\rangle$ the adjoint monad is $\mathbf{S} \rightarrow \mathbf{S}^{\circ}$ with adjunction

$$
J=\left\langle S, S, \mu^{\circ} \cdot \eta, \eta^{\circ} \cdot \mu\right\rangle .
$$

REMARK. In a $2^{\circ}$-category the Frobenius monad $\langle F, \eta, \mu, \epsilon, \delta\rangle$ underlies a *-monad if the comonad $\langle F, \epsilon, \delta\rangle$ equals $\langle F, \eta, \mu\rangle^{\circ}$.

The adjunction $J$ gives rise to a new *-monad

$$
\mathbf{M}(J)=\left\langle S S, \mu^{\circ} \cdot \eta, S\left(\eta^{\circ} \cdot \mu\right) S\right\rangle
$$

related to the original *-monad $\mathbf{S}$ as follows.

Proposition 7. Consider a *-monad $\mathbf{S}$ and its underlying adjoint monad $J: S \dashv \mathbf{S}^{\circ}$. The involute of the multiplication yields a map of monads $\mu^{\circ}$ : $\mathbf{S} \rightarrow \mathbf{M}(J)$. 
Proof. Given the *-monad $S=\langle S, \eta, \mu\rangle$ we show $\mu^{\circ}: S \rightarrow S S$ is compatible with units and with multiplication as diagrammed:
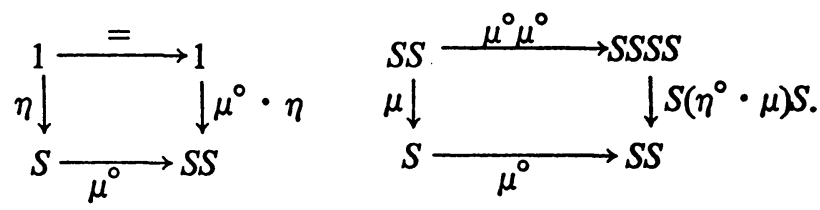

The first diagram is verified by inspection. The second diagram is verified by applying both equations of $(*)$ and the coassociativity of $\mu^{\circ}$.

A conceptual characterization of *-monads is given by the converse.

THEOREM 8. In a $2^{\circ}$-category, if an adjoint monad $J=\langle T, G, n, e\rangle$ : $\mathbf{T} \neg \mathbf{G}$ is

(i) self-dual: $J^{\circ}=\left\langle G, T, e^{\circ}, n^{\circ}\right\rangle: \mathbf{G}^{\circ} \dashv \mathbf{T}^{\circ}$ equals $J$ : $\mathbf{T} \dashv \mathbf{G}$ and

(ii) $\mu^{\circ}: T \rightarrow T T$ yields a map of monads $\mathrm{T} \rightarrow \mathrm{M}(J)$,

then $\mathrm{T}$ is $a *$-monad and $\mathrm{J}: \mathrm{T} \dashv \mathrm{G}$ is its underlying adjoint monad.

PrOOF. By compatibility (ii) of units $n=\mu^{\circ} \cdot \eta$ holds, hence by (i) we have $e=n^{\circ}=\eta^{\circ} \cdot \mu$. To verify the first equation of (*) compose $\eta^{\circ} T$ with both sides of the compatibility (ii) equation for multiplications, then simplify. Verify the second equation of (*) similarly.

An adjoint monad in $T$ is just a monad in the 2-category $J(T)$ of adjunctions [5, p. 136], while monad functors in $T$ are $-T$ squares, which "lift to adjoint squares" if the monads have right adjoints $[5$, p. 135]. The construction of $\operatorname{Mnd}(T)$ can be lifted over the monads with right adjoints to a $2^{\circ}$-category $\operatorname{AdjMnd}(T)$ which will contain $* \operatorname{Mnd}(T)$ as those 0 -cells and 1-cells fixed under the involution extending that of $\S 4$.

6. Examples of *-monadic adjunctions. An adjunction in a $2^{\circ}$-category is *-monadic if the comparison 1-cell to the corresponding terminal realization is an isomorphism. In this situation we also say the right adjoint is *-monadic. For example, as in the introduction, let $\mathbf{E}(\mathbf{R})$ denote the category of linear transformations between finite dimensional Euclidean spaces over the real numbers $\mathbf{R}$. Then $* \mathrm{E}(\mathrm{R})$ is a *-category where the involution takes an $R$-linear map $f: X \rightarrow Y$ into its adjoint map $f^{*}: Y \rightarrow X$ which is related by the inner product equation

$$
(f x \mid y)=\left(x \mid f^{*} y\right) \text { for all }(x, y) \in X \times Y .
$$

Similarly, the complex numbers $\mathbf{C}$ and the quaternions $\mathbf{H}$ yield *-categories of Euclidean vector spaces. The two primary forgetful functors and their composite functor $* \mathrm{E}(\mathrm{H}) \rightarrow * \mathrm{E}(\mathrm{C}) \rightarrow * \mathrm{E}(\mathrm{R})$ are $*$-monadic in $*$ Cat; moreover, so are their left adjoints. 
Given a finite group $G$, the construction of Proposition 5 yields a *-monad on $* E(\Lambda)$ for $\Lambda=R, C$ or $H$. Thus, by Theorem 2 each has a *-category $* E(\Lambda)^{G}$ of *-algebras. In particular, $* \mathrm{E}(C)^{G}$ is the category of intertwining operators (=G-module maps) between (finite dimensional) unitary representations of $G$. If $H$ is a subgroup' of $G$ and if $\Lambda_{1}$ is $R, C$, or $H$, then there is a natural *-monadic functor $* \mathrm{E}(\Lambda)^{G} \rightarrow * \mathrm{E}\left(\Lambda_{1}\right)^{H}$; moreover, its (left) adjoint is *-monadic, too. With $G=H=1$ the trivial group, we have the statements of the previous paragraph. With $\Lambda=\Lambda_{1}=C$, the classical situation of induced representations occurs.

These examples generalize to bounded linear transformations between infinite dimensional Hilbert spaces, to maps between fiber bundles (with fibers as above) over compact Hausdorff spaces, to residuated maps between orthomodular lattices, and to other *-functor categories.

These motivating examples are studied in [6].

\section{REFERENCES}

1: S. Eilenberg and J. C. Moore, Adjoint functors and triples, Illinois J. Math. 9 (1965), 381-398. MR 32 \#2455.

2. H. Kleisli, Every standard construction is induced by a pair of adjoint functors, Proc. Amer. Math. Soc. 16 (1965), 544-546. MR 31 \#1289.

3. F. W. Lawvere, Ordinal sums and equational doctrines, Seminar on Triples and Categorical Homology Theory, Lecture Notes in Math., vol. 80, Springer-Verlag, New York, 1969, pp. 141-155. MR 39 \#1512. 1971.

4. S. MacLane, Categories for the working mathematician, Springer-Verlag, New York,

5. P. H. Palmquist, The double category of adjoint squares, Reports of the Midwest Category Seminar, V, Lecture Notes in Math., vol. 195,Springer-Verlag, New York, 1971. MR 44 \#6788.

6. - Baer *-categories (in preparation).

7. R. Street, The formal theory of monads, J. Pure Appl. Algebra 2 (1972), 149-168. MR 45 \#8701.

DEPARTMENT OF MATHEMATICS, HARVEY MUDD COLLEGE, CLAREMONT, CALIFORNIA 91711

Current address: 4545 Sandburg Way, Irvine, California 92664 